\title{
SPIRAL ARMS IN GRAVITATIONALLY UNSTABLE PROTOPLANETARY DISKS AS IMAGED IN SCATTERED LIGHT
}

\author{
Ruobing Dong (董若冰 $)^{1,2,4}$, Cassandra Hall ${ }^{3}, \mathrm{Ken} \mathrm{Rice}^{3}$, ANd Eugene Chiang (蒋诒曾 $)^{2}$ \\ ${ }^{1}$ Nuclear Science Division, Lawrence Berkeley National Lab, Berkeley, CA 94720, USA; rdong2013@berkeley.edu \\ ${ }_{3}^{2}$ Department of Astronomy, University of California at Berkeley, Berkeley, CA 94720, USA \\ ${ }^{3}$ Institute for Astronomy, University of Edinburgh, Blackford Hill, Edinburgh EH9 3HJ, UK \\ Received 2015 September 4; accepted 2015 September 30; published 2015 October 19
}

\begin{abstract}
Combining 3D smoothed-particle hydrodynamics and Monte Carlo radiative transfer calculations, we examine the morphology of spiral density waves induced by gravitational instability (GI) in protoplanetary disks, as they would appear in direct images at near-infrared (NIR) wavelengths. We find that systems with disk-to-star-mass ratios $q=M_{\text {disk }} / M_{\star}$ that are $\sim 0.25$ or more may produce prominent spiral arms in NIR imaging, remarkably resembling features observed in the MWC 758 and SAO 206462 systems. The contrast of GI-induced arms at NIR wavelengths can reach a factor of $\sim 3$, and their pitch angles are about $10^{\circ}-15^{\circ}$. The dominant azimuthal wavenumber of GI-induced spiral arms roughly obeys $m \sim 1 / q$ in the range $2 \lesssim 1 / q \lesssim 8$. In particular, a massive disk with $q \approx 0.5$ can exhibit grand-design $m=2$ spirals. GI-induced arms are in approximate corotation with the local disk, and may therefore trap dust particles by pressure drag. Although GI can produce NIR spiral arms with morphologies, contrasts, and pitch angles similar to those reported in recent observations, it also makes other demands that may or may not be satisfied in any given system. A GI origin requires that the spirals be relatively compact, on scales $\lesssim 100 \mathrm{AU}$; that the disk be massive, $q \gtrsim 0.25$; and that the accretion rate $\dot{M}_{\star}$ be high, on the order of $10^{-6} M_{\odot} \mathrm{yr}^{-1}$.
\end{abstract}

Key words: circumstellar matter - planets and satellites: formation - protoplanetary disks - stars: formation stars: pre-main sequence - stars: variables: T Tauri, Herbig Ae/Be

\section{INTRODUCTION}

In the past few years, direct imaging at near-infrared (NIR) wavelengths has revealed a panoply of fine features in gaseous protoplanetary disks, thanks to the commissioning of several major high contrast, high angular resolution, adaptive optics imaging instruments onboard 8-m class telescopes (e.g., Subaru/HiCIAO, Suzuki et al. 2010; Very Large Telescope (VLT)/SPHERE, Beuzit et al. 2008; Gemini/GPI, Macintosh et al. 2008). These observations image starlight scattered by dust grains in disk surface layers, and they take advantage of polarized intensity (PI) imaging (e.g., Perrin et al. 2004; Hinkley et al. 2009) to achieve inner working angles as small as 0 ". 1 (corresponding to $\sim 14 \mathrm{AU}$ at the distance of Taurus) with sub- 0 "' 1 angular resolution. The various kinds of radial and azimuthal structures revealed promise to shed light on planet formation processes in disks.

One particularly interesting discovery from NIR disk imaging surveys is the large scale spiral-arm-like features identified in two protoplanetary disks: MWC 758 (Benisty et al. 2015; see also Grady et al. 2013) and SAO 206462 (Garufi et al. 2013; see also Muto et al. 2012). The primary in both cases is a Herbig star $\left(1.8 M_{\odot}\right.$ in MWC 758, Chapillon et al. 2008; and 1.6 $M_{\odot}$ in SAO 206462, Andrews et al. 2011), and both systems are recognized as transitional disks with a giant depleted central region (Andrews et al. 2011; Pérez et al. 2014; Marino et al. 2015). The two major arms in each system are in nearly $m=2$ rotational symmetry, and are located at tens of to $\sim 100 \mathrm{AU}$ from the center. The arms can be easily traced over at least $180^{\circ}$ in the azimuthal direction, and they are quite open with large pitch angles (defined as the angle between the azimuthal direction and the tangent of the arm).

\footnotetext{
${ }^{4}$ NASA Hubble Fellow.
}

Besides MWC 758 and SAO 206462, spiral arms on smaller azimuthal (and/or radial) scale have been discovered in a number of systems, such as AB Aur (Hashimoto et al. 2011), HD 142527 (Fukagawa et al. 2006; Canovas et al. 2013; Avenhaus et al. 2014), and HD 100546 (Currie et al. 2014). The number of arms in this second class of system is generally more than 5 (with the exception of HD 100546, which exhibits a couple of small arms), and the arms have an azimuthal extension generally no bigger than $90^{\circ}$.

Recently, Dong et al. (2015a) have shown that $m=2 \mathrm{arms}$ such as the pair in MWC 758 may be explained by density waves excited by one giant companion (planet or brown dwarf) on the periphery of the arms (see also Zhu et al. 2015). In this paper, we investigate another physical mechanism that is also able to excite density waves in disks - gravitational instability (GI; e.g., Rice et al. 2003; Lodato \& Rice 2004, 2005). We focus on the observational signatures of GI-induced density waves in NIR imaging observations, as predicted by 3D smoothed-particle hydrodynamics (SPH) + Monte Carlo radiative transfer (MCRT) simulations. We ask specifically whether GI-induced features can resemble the observed spiral arms. This general topic has been explored by Dipierro et al. (2015a), who studied the evolution of dust in a fixed gas disk background obtained from the Lodato \& Rice (2004) SPH GI simulations. They calculated 2D dust surface density distributions, which were then puffed up in the vertical direction by a parametrized gaussian profile and fed into a 3D MCRT code to produce synthetic images. These authors focused on relatively low mass disks, computing spatial distributions of grains with different size distributions and simulating both NIR scattered light and mm-wave ALMA images (see also an earlier paper by Dipierro et al. 2014 that treated gas and dust as well-mixed and that concentrated on ALMA observations). By comparison, we 
Table 1

Model Properties

\begin{tabular}{lcccccccc}
\hline \hline Model & $q=M_{\text {disk }} / M_{\star}$ & $\begin{array}{c}\dot{M}_{\star} \\
\left(M_{\odot} \mathrm{yr}^{-1}\right)\end{array}$ & Dominant Azimuthal Number & $\Omega_{\mathrm{p}}{ }^{\mathrm{a}}$ & $\begin{array}{c}r_{\mathrm{CR}}{ }^{\mathrm{b}} \\
(\mathrm{AU})\end{array}$ & $\begin{array}{c}\sum_{\text {peak }} / \Sigma_{\mathrm{b}}{ }^{\mathrm{c}} \\
I_{\text {peak }} / I_{\mathrm{b}}{ }^{\mathrm{d}}\end{array}$ & $\begin{array}{c}\text { Pitch Angle }^{\mathrm{e}} \\
\left({ }^{\circ}\right)\end{array}$ \\
\hline MD0125 & 0.125 & $7 \times 10^{-8}$ & $m \sim 4-8$ & $\sim 2 \pi / 400 \mathrm{yr}$ & 56 & {$[1.34,1.60]$} & {$[1.06,1.50]$} \\
MD025 & 0.25 & $5 \times 10^{-7}$ & $m \sim 4$ & $\sim 2 \pi / 310 \mathrm{yr}$ & 49 & {$[3.48,2.28]$} & {$[2.10,1.83]$} & 10 \\
MD05 & 0.5 & $5 \times 10^{-6}$ & $m=2$ & $\sim 2 \pi / 280 \mathrm{yr}$ & 47 & {$[2.50,3.20]$} & {$[1.71,2.87]$} & 15 \\
\hline
\end{tabular}

Notes.

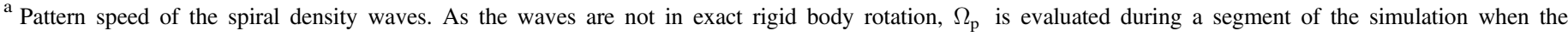
morphologies of the waves did not appear to evolve much by eye.

${ }^{\mathrm{b}}$ Corotation radius at which $\Omega_{\text {Keplerian }}\left(r_{\mathrm{CR}}\right)=\Omega_{\mathrm{p}}$.

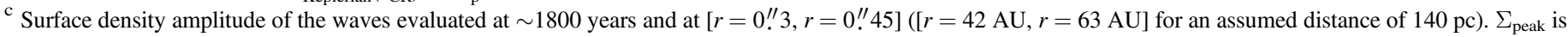
the peak surface density; $\Sigma_{\mathrm{b}}$ is the background surface density taken to be the azimuthal average at the same radius.

d Same as (c), but for scattered light intensity in convolved images.

e Averaged pitch angle of the arms in convolved images.

will focus on NIR imaging, and will explore a larger range of disk masses, making direct comparisons between models and scattered light observations of real astronomical sources. Pohl et al. (2015) combined 2D hydro and 3D MCRT simulations to study the appearance of spiral arms at NIR wavelengths in GI disks with the added complication of an embedded planet. Our aim here is to isolate the effects of GI so that we can compare planet versus GI-based interpretations of NIR images.

The structure of our paper is as follows. The hydro and MCRT simulations are introduced in Section 2; the modeling results are presented in Section 3; and a summary and connections to observations are given in Section 4.

\section{SPH AND MCRT SIMULATIONS}

The general workflow of our modeling is as follows. We carry out 3D global SPH simulations to calculate the gas density structures of gravitationally unstable disks (Section 2.1). The resulting models are read into the Whitney et al. (2013) 3D dust MCRT code to produce synthetic model images at $1.6 \mu \mathrm{m}$ ( $H$ band; Section 2.2), assuming that dust is uniformly mixed with gas. The vertical structure of the SPH disk is iterated in the MCRT code until radiative equilibrium and hydrostatic equilibrium (HSEQ) are achieved. Finally, synthetic images are convolved by a Gaussian point-spread function (PSF) to achieve an angular resolution comparable to recent observations.

\subsection{SPH Simulations}

The SPH simulations are based on the code developed by Bate et al. (1995), augmented by a hybrid radiative transfer formalism that combines the polytropic approximation with the flux-limited diffusion approximation (Forgan et al. 2009; Stamatellos \& Whitworth 2009) to model both global cooling and radiative transfer. The opacity and temperature of the gas are calculated using an equation of state that accounts for the effects of $\mathrm{H}_{2}$ dissociation, $\mathrm{H}^{0}$ ionization, $\mathrm{He}^{0}$ and $\mathrm{He}^{+}$ ionization, ice evaporation, dust sublimation, molecular absorption, bound-free and free-free transitions and electron scattering (Bell \& Lin 1994; Boley et al. 2007; Stamatellos et al. 2007). The simulations include heating of the disk through $P d V$ work and shocks; stellar irradiation is not included.

The mass of the central central star $M_{\star}$ is assumed to be 1 $M_{\odot}$. In total we construct three models (Table 1) where the disk-to-star-mass ratio $q=M_{\text {disk }} / M_{\star}$ equals $0.125,0.25$, and
0.5 , respectively. Each gas disk comprises $2 \times 10^{6}$ SPH particles, initially located between 10 and $60 \mathrm{AU}$, and distributed in such a way that the initial surface density profile $\Sigma_{0} \propto r^{-3 / 2}$ and the initial sound speed profile $c_{\mathrm{s}} \propto r^{-1 / 4}$. By limiting the initial disk to be within $60 \mathrm{AU}$, we ensure that these models remain quasi-steady, rather than fragmenting into bound companions (e.g., Rafikov 2005; Stamatellos et al. 2007; Clarke 2009; Rice \& Armitage 2009). The accretion rate of the disks $\left(\dot{M}_{\star}\right.$ in the table $)$ is calculated by counting the rate at which particles accrete onto the star. All simulations are run for about 3500 years, or $\sim 10$ orbits at $50 \mathrm{AU}$ where the spiral arms are most prominent.

\subsection{MCRT Simulations}

Density structures obtained in our SPH simulations are postprocessed using the Whitney MCRT code. We construct a 3D disk in spherical coordinates, with $400 \times 200 \times 256$ cells in $r$ (radial, from 5 to $100 \mathrm{AU}$ with logarithmic spacing to better resolve the inner disk), $\theta$ (polar, from 0 to $\pi$ with power law spacing to better resolve the disk midplane at $\theta=\pi / 2$ ), and $\phi$ (azimuthal, from 0 to $2 \pi$ with uniform spacing). Photons from an $R_{\star}=2 R_{\odot}$ and $T_{\star}=4500 \mathrm{~K}$ central star (corresponding to a $1 M_{\odot}$ star that is 2 Myr old on the Hayashi track; Hayashi 1961) are absorbed/reemitted or scattered by dust in the surrounding disk. ${ }^{5}$ The temperature in each grid cell is calculated based on the radiative equilibrium algorithm described in Lucy (1999). The power released from accretion $\dot{M}_{\star}$ at each radius is deposited locally into the cells, and the accretion power between the magnetic truncation radius (assumed to be at $5 R_{\star}$ ) and the stellar surface is added to the total luminosity of the star. The scattering phase function is approximated using the Henyey \& Greenstein (1941) function. Polarization is calculated assuming a Rayleigh-like phase function for the linear polarization (White 1979). All simulations are run with 1 billion photon packets.

Our MCRT simulations are run with the "HSEQ" switch in the Whitney code turned on. In some of our SPH simulations, the temperature in the outer disk reaches a minimum floor (artificially set in the SPH code) that is lower than what is estimated using the MCRT calculations. As mentioned in Section 2.1, we do not have stellar irradiation in the

\footnotetext{
5 We also experimented with a $1.8 M_{\odot}$ pre-main-sequence star (as in the MWC 758 system; Chapillon et al. 2008) in the MCRT simulations, and found that the resulting scattered light images merely brightened by a factor of $\sim 4$ because of the higher stellar luminosity.
} 


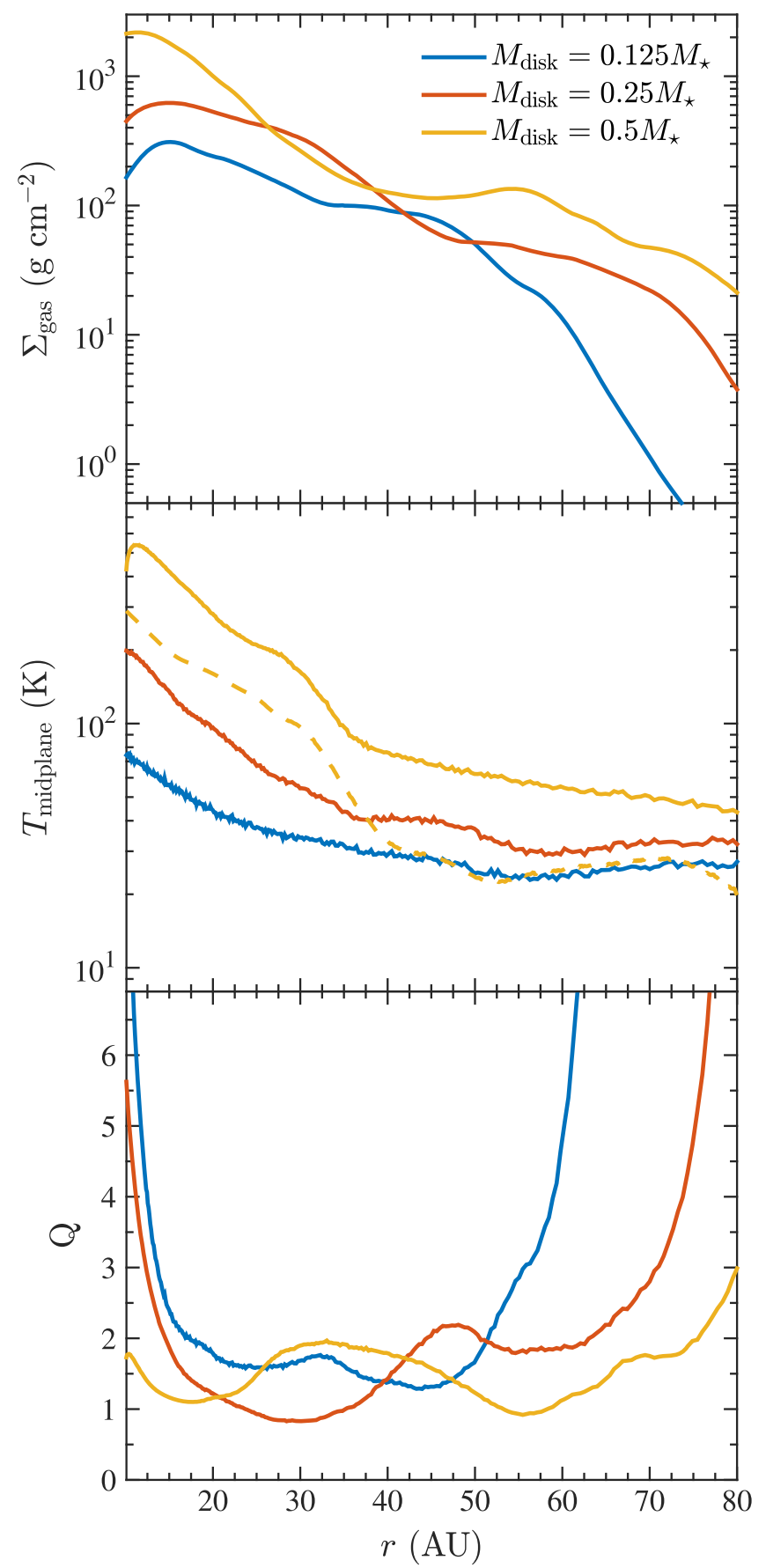

Figure 1. Surface density (top, from SPH simulations), midplane temperature (middle, from MCRT simulations), and Toomre $Q$ (bottom, Equation (2)) for our models of various disk masses. The dashed curve in the middle panel is the midplane temperature from the SPH simulation for the $M_{\text {disk }}=0.5 M_{\star}$ model. The MCRT temperature can be up to a factor of $\sim 2$ higher than the SPH temperature in the outer disk.

SPH simulations. As a result, the outer regions of our SPH disks can be artificially cold, and thin in the vertical direction. To correct this effect, we readjust the vertical density distribution of the disks in the MCRT simulations using the MCRT temperature and not the SPH temperature, while still using the surface density distribution from the SPH simulations. This inconsistency of method is not serious; if we were to use a higher temperature in the SPH simulations, the spiral structures would be only slightly weaker than suggested here (Rice et al. 2011; Rafikov 2015) for fixed disk mass; thus the $q$-values reported here are slightly underestimated.

In MCRT calculations, the vertical structure is solved based on a self-consistent HSEQ condition, as gas pressure balances gravity in the vertical direction $z$ :

$$
\frac{d p}{d z}=-\rho \frac{z}{r} \frac{G M(r)}{r^{2}}
$$

where $p$ is pressure, $\rho$ is density, and $M(r)=M_{\star}+M_{\text {disk }}(r)$ with $M_{\text {disk }}(r)$ equal to the total disk mass within $r$. The quantities $T$ and $\rho$ are iterated until convergence is achieved.

The dust grains in the disk are assumed to be interstellar medium (ISM) grains (Kim et al. 1994) made of silicate, graphite, and amorphous carbon. Their size distribution is a smooth power law in the range of $0.02-0.25 \mu \mathrm{m}$ followed by an exponential cut off beyond $0.25 \mu \mathrm{m}$. These grains are small enough to be dynamically well coupled to the gas, so that their volume density $\rho_{\text {dust }}$ is linearly proportional to the gas $\rho_{\text {gas }}$; we take the ratio to be $\rho_{\text {gas }}: \rho_{\text {dust }}=1000: 1 .^{6}$ The optical properties of the grains can be found in Figure 2 of Dong et al. (2012).

Full resolution synthesized PI images at $H$ band $(1.6 \mu \mathrm{m})$ are produced from MCRT simulations, ${ }^{7}$ and then convolved by a Gaussian PSF with a full width half maximum (FWHM) of 0 ". 05 assuming a distance of $140 \mathrm{pc}$, to achieve an angular resolution comparable to those obtained with Subaru, VLT, and Gemini (the FWHM of a theoretical Airy disk is $1.028 \lambda / D \sim$ 0 ." 04 for a primary mirror with a diameter $D=8.2 \mathrm{~m}$ at $\lambda=1.6 \mu \mathrm{m})$.

\section{RESULTS}

The radial profiles of the surface density, midplane temperature, and Toomre $Q$ for all models are shown in Figure 1. GI develops in all cases, and manifests itself in the form of spiral density waves. Disks become marginally gravitationally unstable when

$$
Q=\frac{c_{\mathrm{s}} \kappa}{\pi \mathrm{G} \Sigma_{\mathrm{gas}}}
$$

is $\lesssim 2$, where $\kappa$ is the epicyclic frequency. As $q$ increases, the $Q$ $\lesssim 2$ regime expands, from $\sim 20-50 \mathrm{AU}$ in $\mathrm{MD} 0125$, to $\sim 20-60$ AU in MD025, and to 10-70 AU in MD05.

As described in Lodato \& Rice (2005), GI disks in our models $(q \leqslant 0.5)$ undergo three phases: $(1)$ the initial settling phase that lasts for about 500 years, during which the disk adjusts its outer radius, and material redistributes radially by axisymmetric evolution and ring formation; (2) the "burst" phase that lasts for about 1000 years, during which spiral density waves form and grow; and (3) the asymptotic phase, where GI is self-regulated and the disk reaches a quasiequilibrium state. In the latter two phases, the dominant azimuthal wavenumber $m$ (Table 1) decreases as $q$ increases, consistent with Lodato \& Rice (2004) and Cossins et al. (2009). In our $q$ range, we find $m \sim 1 / q$, with the approximation

\footnotetext{
6 This corresponds to a conventional 100:1 gas-to-solid mass ratio and a 10:1 solid-to-ISM-dust mass ratio. The remaining $90 \%$ of the solids are assumed to be in the form of large grains and/or planetesimals that may have settled to the disk midplane and do not affect NIR scattering.

7 In this work, the physical quantity recorded in all model images is the specific intensity in units of $\left(\mathrm{mJy} \operatorname{arcsec}{ }^{-2}\right)$, or $\left(10^{-26} \mathrm{erg} \mathrm{s}^{-1} \mathrm{~cm}^{-2} \mathrm{~Hz}^{-1} \operatorname{arcsec}^{-2}\right)$.
} 


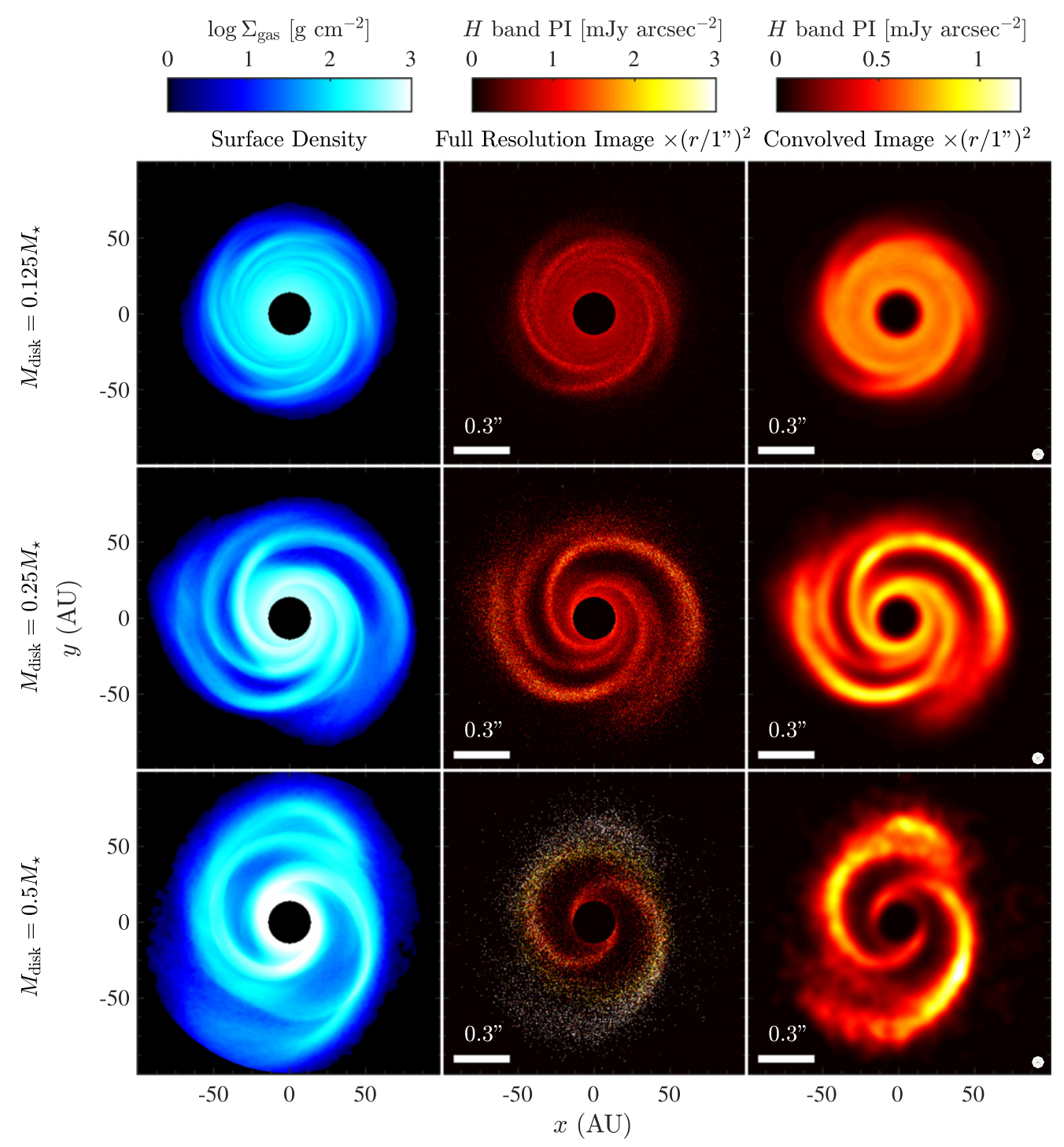

Figure 2. Surface density and $H$ band PI images at face-on viewing angles (scaled by $r^{2}$ ) and at $\sim 1800$ years of the models at 140 pc. Systems are assumed to be at $140 \mathrm{pc}$. Convolved images (right column) are full resolution MCRT images (middle column) convolved by a Gaussian PSF with a FWHM of 0."05 (marked at the lower right corner). The inner $14 \mathrm{AU}(0$ !' 1$)$ in all panels is masked out.

becoming less accurate at low $q$ values $(m$ does not increase as fast as $1 / q$ ).

The spiral density waves have a pattern speed $\Omega_{\mathrm{p}}$, listed in Table 1 along with the corresponding corotation radius $r_{\mathrm{CR}}$ $\left(\Omega_{\text {Keplerian }}\left(r_{\mathrm{CR}}\right)=\Omega_{\mathrm{p}}\right)$. In general, the spirals are roughly in Keplerian rotation, and $r_{\mathrm{CR}}$ coincides with the main part of the arms. This is consistent with Cossins et al. (2009), who found for a $q=0.125$ disk that $\Omega_{\mathrm{p}}$ differs from the local $\Omega_{\text {Keplerian }}$ by $\lesssim 15 \%$. This property should lead to dust trapping by the arms and promote grain growth and planetesimal formation. As shown by Rice et al. (2006) and Paardekooper \& Mellema (2006), dust particles with Stokes number $\sim 1$ tend to be trapped at local pressure maxima in disks; this has been demonstrated both in radial (e.g., Pinilla et al. 2012; Zhu et al. 2012; de Juan Ovelar et al. 2013; Dong et al. 2015b) and azimuthal gas structures (e.g., Lyra \& Lin 2013; Zhu et al. 2014; Mittal \& Chiang 2015) as a mechanism to explain observed concentrations of dust particles in ALMA disks (e.g., Casassus et al. 2013; Fukagawa et al. 2013; van der Marel et al. 2013; Pérez et al. 2014; van der Marel et al. 2015). In the case of spiral arms, Rice et al. (2004) have shown that for $q=0.25$, spiral density waves can act to trap dust particles, and through 2D shearing box simulations, Gibbons et al. (2012) concluded that the local dust surface density can be enhanced by up to $\sim 3.5$ orders of magnitude relative to the gas.

The surface density and synthetic $H$ band images are shown in Figure 2, and the radial as well as azimuthal profiles of the convolved images are shown in Figure 3. In $\Sigma_{\text {gas }}$, spiral density waves are clearly present in all cases, while their amplitudes increase from a factor of $\sim 1.5$ in MD0125 to a factor of $\sim 3$ in $\operatorname{MD05}\left(\Sigma_{\text {peak }} / \Sigma_{\mathrm{b}}\right.$ in Table 1$)$. In the convolved images, only MD025 and MD05 show prominent spiral patterns, while the arms in MD0125 are weak and hard to distinguish from the background. In particular, the MD05 model shows a pair of grand-design $m=2 \mathrm{arms}$, with a morphology very similar to the pair in the MWC 758 system. The contrast of the arms to the background $\left(I_{\text {peak }} / I_{\mathrm{b}}\right.$ in Table 1$)$ ranges from a factor of $\sim 2$ in MD025 to a factor of $\sim 3$ in MD05. Note that our arm contrast in convolved images should be taken as upper limits, as realistic instrumental effects such as flux loss and 


\section{Radial Profile of Convolved Images}

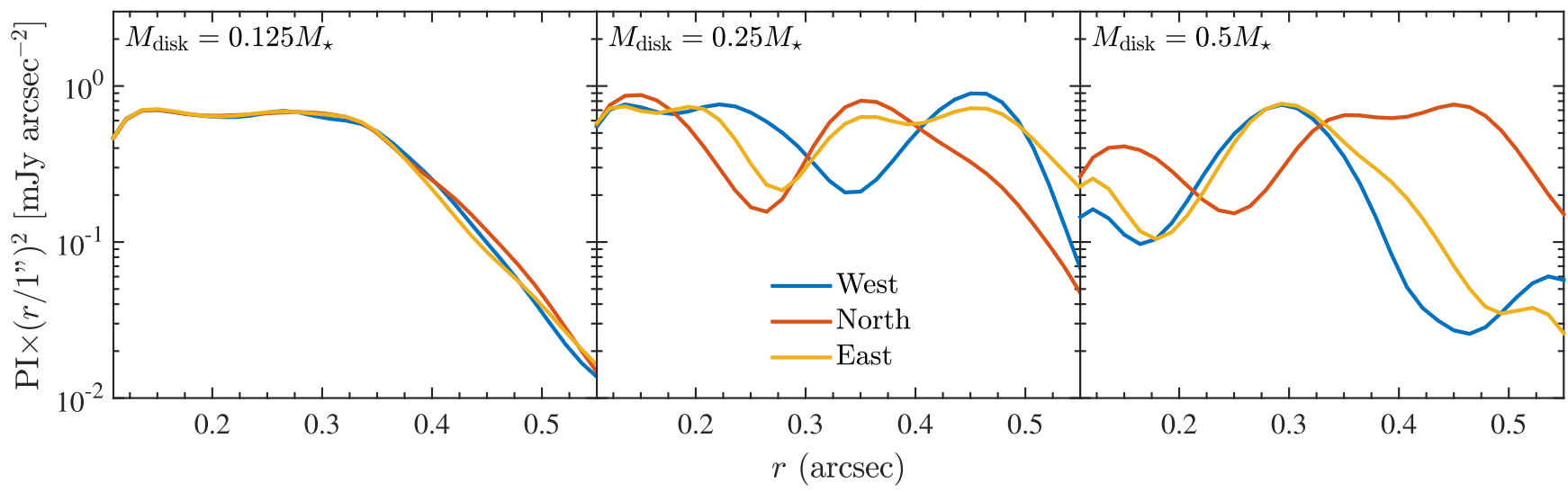

Azimuthal Profile of Convolved Images

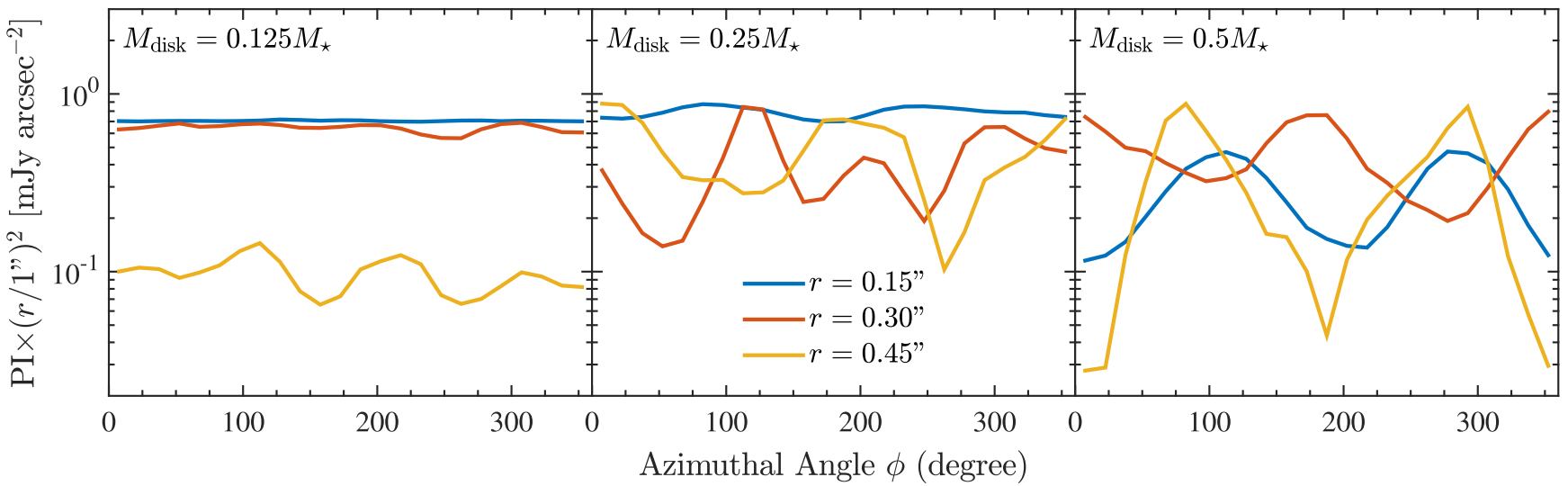

Figure 3. Radial profiles in the east, north, and west directions (top) and azimuthal profiles at $r=0$ !. 15,0 !. 3 , and 0 ! 45 (bottom) of the $r^{2}$-scaled convolved images in Figure 2. The peaks on all curves indicate interceptions with the spiral arms. In the azimuthal profiles, the double-peak pattern (with the two peaks separated by $\sim 180^{\circ}$ ) is prominent in MD05 at all radii, indicating the dominance of the $m=2$ mode; the quadrupole-peak pattern (with the peaks separated by $\sim 90^{\circ}$ ) is present but less prominent in MD025; the MD0125 model only shows a quadrupole-peak pattern at large radius and low amplitude arms at smaller radius.

observational noise, which may affect both the absolute and relative fluxes, are not included in the production of our model images (beyond the intrinsic Monte Carlo noise in the radiative transfer images). The pitch angle of the arms (Table 1) increases from $10^{\circ}$ in MD0125 to $15^{\circ}$ in MD05, values that are consistent with those characterizing the SAO 206462 and MWC 758 systems (Dong et al. 2015a).

\section{SUMMARY AND CONNECTION WITH OBSERVATIONS}

We studied the observational signatures of GI-induced spiral arms in direct imaging observations at NIR wavelengths with 3D SPH calculations and 3D MCRT simulations. Our main conclusions are:

1. GI can produce prominent spiral arms in NIR scattered light images when $q=M_{\text {disk }} / M_{\star} \gtrsim 0.25$. Their morphologies (see Figure 4) resemble strongly the ones discovered in recent observations (e.g., SAO 206462 as imaged by Garufi et al. 2013, and most strikingly, the pair of $m=2$ arms in MWC 758 found by Benisty et al. 2015).

2. When $q \lesssim 0.1$, GI-induced spiral arms are small in size and weak in scattered light images, with contrasts $\lesssim 1.5$. They may be difficult to detect with the current angular resolution of NIR observations. A similar conclusion was reached by Dipierro et al. (2015a).

3. The dominant azimuthal wavenumber of GI-induced spiral arms is $m \sim 1 / q$ in the range of $2 \lesssim 1 / q \lesssim 8$.

4. The spiral arms have a pattern speed that roughly agrees with the Keplerian speed at where the arms are. Dust trapping by arms at local pressure maxima may therefore be possible.

Although qualitatively our $q=0.5$ and $q=0.25$ model images show good agreement with the MWC 758 and SAO 206462 observed images (Figure 4), whether GI is actually at work in these two systems is unclear. There are at least three potential issues that need to be addressed to validate GI as a possible explanation for these systems.

1. Our set of three models shows that $q$ has to be $\gtrsim 0.25$ for the spiral density waves to be prominent in scattered light images as seen in observations (in particular, granddesign $m=2$ arms require $q \approx 0.5$ ). However, under conventional assumptions for dust opacity and dust-togas-mass ratio, Andrews et al. (2011) estimated the disk mass $M_{\text {disk }}$ of these two systems using mm continuum observations, and concluded that $q$ is on the order of $1 \%$ in both cases. The caveat, of course, is that the conventional assumptions for dust opacity and dust-togas-mass ratio may not be correct, and $M_{\text {disk }}$ may have 

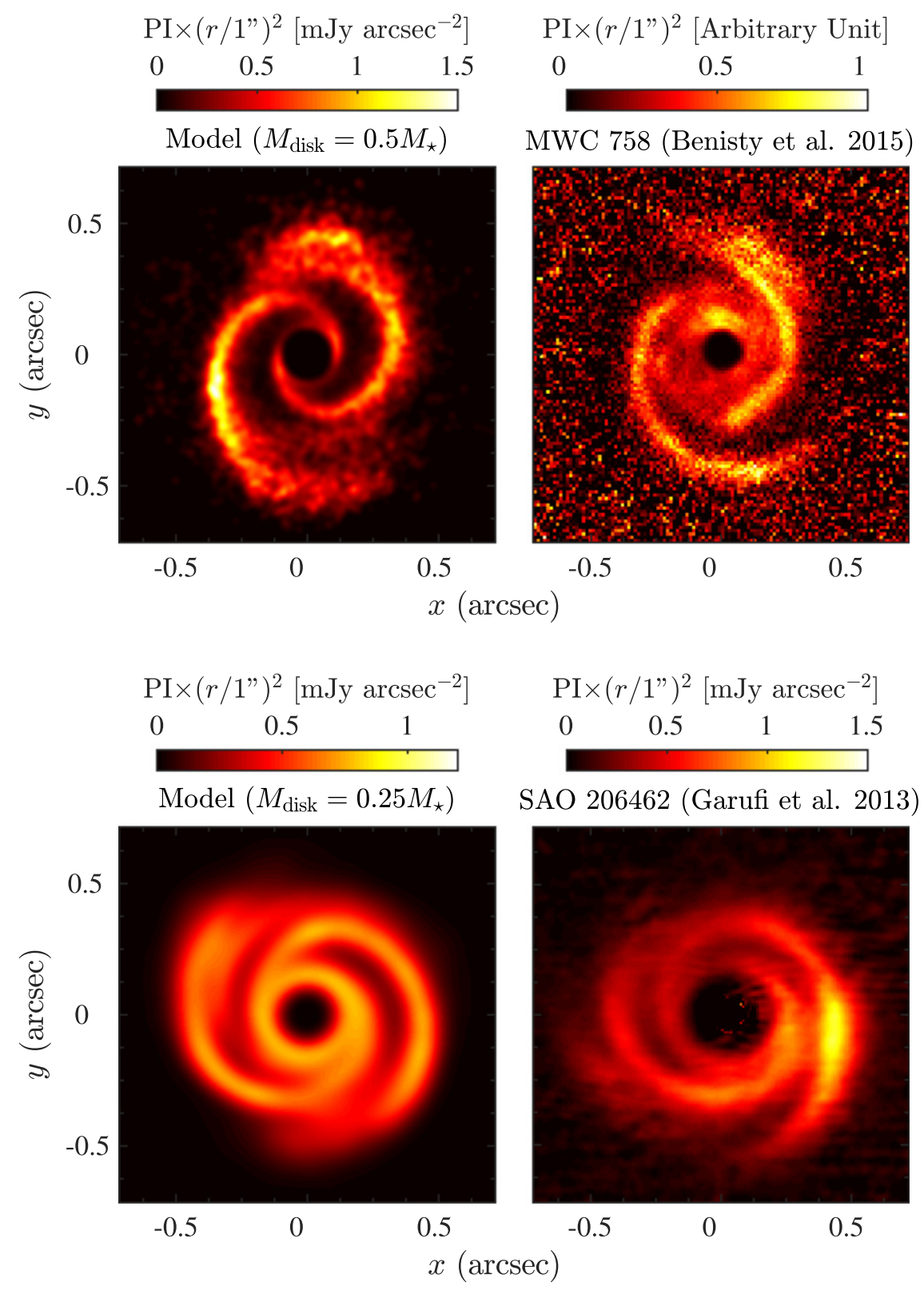

Figure 4. Comparisons of the $q=0.5$ model with MWC 758 (Benisty et al. 2015, top), and the $q=0.25$ model with SAO 206462 (Garufi et al. 2013 , bottom). The models are assumed to be at $140 \mathrm{pc}$, and convolved by a Gaussian PSF with FWHM =0!.03 (MD05) and FWHM = 0!.09 (MD025) to match the corresponding observations. The units in all images are mJy arcsec ${ }^{-2}$, except for MWC 758 for which the units are arbitrary.

been underestimated, at least in some cases. See our discussion on the HL Tau system below. On the other hand, disks with $q \gtrsim 0.25$ may develop substantial nonKeplerian velocity components, the detections of which will help determine the masses of these systems (Rosenfeld et al. 2014).

2. The measured stellar mass accretion rates of the two systems are both on the order of $10^{-8} M_{\odot} \mathrm{yr}^{-1}$ (Eisner et al. 2009; Grady et al. 2009), about two orders of magnitude smaller than the $\dot{M}_{\star}$ 's from our models MD025 and MD05. The caveat here is that accretion in GI disks can be highly episodic. As shown in Vorobyov \& Basu (2015) (their Figure 3), although the timeaveraged $\dot{M}_{\star}$ can reach $\sim 10^{-6} M_{\odot} \mathrm{yr}^{-1}, \dot{M}_{\star}$ fluctuates with large amplitude and can occasionally dip under $10^{-8} M_{\odot} \mathrm{yr}^{-1}$.
3. While the spiral arms in SAO 206462 are located at $\sim 30-80$ AU from the star, consistent with our models, the $m=2$ arms in MWC 758 extend to $\sim 135$ AU, which is formally outside the region that we simulate (MWC 758 is 280 pc away, van Leeuwen 2007; SAO 206462 is 140 pc away, Müller et al. 2011; our model distances are $140 \mathrm{pc})$. GI disks tend to fragment outside a critical radius $r_{\mathrm{f}}$ if the opacity has the form of $\kappa \propto T^{2}$, as expected from the ice-grain-dominated opacity at low temperatures (Rafikov 2005; Rice \& Armitage 2009; Forgan \& Rice 2011, 2013). Both theoretical estimates (Clarke 2009; Rafikov 2009; Kratter et al. 2010) and some numerical simulations (e.g., Stamatellos et al. 2007) have suggested $r_{\mathrm{f}}$ to be around $\sim 100$ AU (but see also Figure 2 in Vorobyov 2013 and Figure 1 in Vorobyov \& Basu 2015, showing spiral arms but no fragments at several 
hundred AU). The radial scale of MWC 758's arms appear dangerously close to, if not exceeding, the fragmentation radius.

We conclude from the above three concerns that it is unclear whether the major spiral arm systems discovered so far from direct NIR imaging surveys can be attributed to GI. Planets, as shown by Dong et al. (2015a), represent a more plausible explanation. Nevertheless, if future NIR imaging observations find prominent spiral arms in disks (1) with large masses $(q \gtrsim 0.25)$, (2) with high stellar accretion rates $\left(\dot{M}_{\star} \gtrsim 10^{-6} M_{\odot} \mathrm{yr}^{-1}\right)$, and (3) on relatively compact scales ( $\lesssim 100 \mathrm{AU}$, safely inside the gravitational fragmentation radius), then GI would be securely implicated. Once such systems are found, the number of spirals in the disk, i.e., the dominant azimuthal wavenumber, will be a good diagnostic of the disk's total mass.

We close with a few remarks on the HL Tau system. With a high accretion rate $\left(9 \times 10^{-6} M_{\odot} \mathrm{yr}^{-1}\right.$, Hayashi et al. 1993) and total gas disk mass estimated from $\mathrm{mm}$ continuum observations ( $q \sim 0.25$, Kwon et al. 2011), the HL Tau system should be prone to GI, as suggested by our model MD025, and prominent low $m$-mode spiral arms are expected to form. This picture seems to be incompatible with the ALMA observations (ALMA Partnership et al. 2015) that reveal concentric rings, likely created by planets (Dipierro et al. 2015b; Dong et al. $2015 \mathrm{~b}$ ). The lack of spirals may suggest that previous estimates of HL Tau's disk mass are too high and/or that the stellar mass estimate is too low.

R.D. thanks Cathie Clarke and Roman Rafikov for educating him on the subject of GI. We thank Myriam Benisty and Antonio Garufi for kindly sharing with us the VLT/SPHERE image of MWC 758, and the VLT/NACO image of SAO 206462 , respectively. We are grateful to the anonymous referee for constructive suggestions that improved the quality of the paper. This project is partially supported by NASA through Hubble Fellowship grant HST-HF-51320.01-A (R.D.) awarded by the Space Telescope Science Institute, which is operated by the Association of Universities for Research in Astronomy, Inc., for NASA, under contract NAS 5-26555. E.C. acknowledges support from NASA and the NSF. Numerical calculations were performed on the SAVIO cluster provided by the Berkeley Research Computing program, supported by the UC Berkeley Vice Chancellor for Research and the Berkeley Center for Integrative Planetary Science.

\section{REFERENCES}

ALMA Partnership, Brogan, C. L., Pérez, L. M., et al. 2015, ApJL, 808, L3 Andrews, S. M., Wilner, D. J., Espaillat, C., et al. 2011, ApJ, 732, 42 Avenhaus, H., Quanz, S. P., Schmid, H. M., et al. 2014, ApJ, 781, 87 Bate, M. R., Bonnell, I. A., \& Price, N. M. 1995, MNRAS, 277, 362 Bell, K. R., \& Lin, D. N. C. 1994, ApJ, 427, 987

Benisty, M., Juhasz, A., Boccaletti, A., et al. 2015, A\&A, 578, L6 Beuzit, J.-L., Feldt, M., Dohlen, K., et al. 2008, Proc. SPIE, 7014, 701418 Boley, A. C., Hartquist, T. W., Durisen, R. H., \& Michael, S. 2007, ApJL, 656, L89

Canovas, H., Ménard, F., Hales, A., et al. 2013, A\&A, 556, A123

Casassus, S., van der Plas, G., M, S. P., et al. 2013, Natur, 493, 191

Chapillon, E., Guilloteau, S., Dutrey, A., \& Piétu, V. 2008, A\&A, 488, 565

Clarke, C. J. 2009, MNRAS, 396, 1066

Cossins, P., Lodato, G., \& Clarke, C. J. 2009, MNRAS, 393, 1157

Currie, T., Muto, T., Kudo, T., et al. 2014, ApJL, 796, L30 de Juan Ovelar, M., Min, M., Dominik, C., et al. 2013, A\&A, 560, A111 Dipierro, G., Lodato, G., Testi, L., \& de Gregorio Monsalvo, I. 2014, MNRAS, 444, 1919

Dipierro, G., Pinilla, P., Lodato, G., \& Testi, L. 2015a, MNRAS, 451, 974

Dipierro, G., Price, D., Laibe, G., et al. 2015b, MNRAS, 453, L73

Dong, R., Rafikov, R., Zhu, Z., et al. 2012, ApJ, 750, 161

Dong, R., Zhu, Z., Rafikov, R. R., \& Stone, J. M. 2015a, ApJL, 809, L5

Dong, R., Zhu, Z., \& Whitney, B. 2015b, ApJ, 809, 93

Eisner, J. A., Graham, J. R., Akeson, R. L., \& Najita, J. 2009, ApJ, 692, 309

Forgan, D., \& Rice, K. 2011, MNRAS, 417, 1928

Forgan, D., \& Rice, K. 2013, MNRAS, 430, 2082

Forgan, D., Rice, K., Stamatellos, D., \& Whitworth, A. 2009, MNRAS, 394, 882

Fukagawa, M., Tamura, M., Itoh, Y., et al. 2006, ApJL, 636, L153

Fukagawa, M., Tsukagoshi, T., Momose, M., et al. 2013, PASJ, 65, L14

Garufi, A., Quanz, S. P., Avenhaus, H., et al. 2013, A\&A, 560, A105

Gibbons, P. G., Rice, W. K. M., \& Mamatsashvili, G. R. 2012, MNRAS, 426, 1444

Grady, C. A., Muto, T., Hashimoto, J., et al. 2013, ApJ, 762, 48

Grady, C. A., Schneider, G., Sitko, M. L., et al. 2009, ApJ, 699, 1822

Hashimoto, J., Tamura, M., Muto, T., et al. 2011, ApJL, 729, L17

Hayashi, C. 1961, PASJ, 13, 450

Hayashi, M., Ohashi, N., \& Miyama, S. M. 1993, ApJL, 418, L71

Henyey, L. G., \& Greenstein, J. L. 1941, ApJ, 93, 70

Hinkley, S., Oppenheimer, B. R., Soummer, R., et al. 2009, ApJ, 701, 804

Kim, S.-H., Martin, P. G., \& Hendry, P. D. 1994, ApJ, 422, 164

Kratter, K. M., Murray-Clay, R. A., \& Youdin, A. N. 2010, ApJ, 710, 1375

Kwon, W., Looney, L. W., \& Mundy, L. G. 2011, ApJ, 741, 3

Lodato, G., \& Rice, W. K. M. 2004, MNRAS, 351, 630

Lodato, G., \& Rice, W. K. M. 2005, MNRAS, 358, 1489

Lucy, L. B. 1999, A\&A, 344, 282

Lyra, W., \& Lin, M.-K. 2013, ApJ, 775, 17

Macintosh, B. A., Graham, J. R., Palmer, D. W., et al. 2008, Proc. SPIE, 7015, 701518

Marino, S., Casassus, S., Perez, S., et al. 2015, ApJ, in press (arXiv:1505.06732) Mittal, T., \& Chiang, E. 2015, ApJL, 798, L25

Müller, A., van den Ancker, M. E., Launhardt, R., et al. 2011, A\&A, 530, A85

Muto, T., Grady, C. A., Hashimoto, J., et al. 2012, ApJL, 748, L22

Paardekooper, S.-J., \& Mellema, G. 2006, A\&A, 453, 1129

Pérez, L. M., Isella, A., Carpenter, J. M., \& Chandler, C. J. 2014, ApJL, 783, L13

Perrin, M. D., Graham, J. R., Kalas, P., et al. 2004, Sci, 303, 1345

Pinilla, P., Benisty, M., \& Birnstiel, T. 2012, A\&A, 545, A81

Pohl, A., Pinilla, P., Benisty, M., et al. 2015, MNRAS, 453, 1768

Rafikov, R. R. 2005, ApJL, 621, L69

Rafikov, R. R. 2009, ApJ, 704, 281

Rafikov, R. R. 2015, ApJ, 804, 62

Rice, W. K. M., \& Armitage, P. J. 2009, MNRAS, 396, 2228

Rice, W. K. M., Armitage, P. J., Bate, M. R., \& Bonnell, I. A. 2003, MNRAS, 339, 1025

Rice, W. K. M., Armitage, P. J., Mamatsashvili, G. R., Lodato, G., \& Clarke, C. J. 2011, MNRAS, 418, 1356

Rice, W. K. M., Armitage, P. J., Wood, K., \& Lodato, G. 2006, MNRAS, 373,1619

Rice, W. K. M., Lodato, G., Pringle, J. E., Armitage, P. J., \& Bonnell, I. A. 2004, MNRAS, 355, 543

Rosenfeld, K. A., Chiang, E., \& Andrews, S. M. 2014, ApJ, 782, 62

Stamatellos, D., Hubber, D. A., \& Whitworth, A. P. 2007, MNRAS, 382, L30

Stamatellos, D., \& Whitworth, A. P. 2009, MNRAS, 400, 1563

Suzuki, R., Kudo, T., Hashimoto, J., et al. 2010, Proc. SPIE, 7735, 30

van der Marel, N., van Dishoeck, E. F., Bruderer, S., et al. 2013, Sci, 340, 1199

van der Marel, N., van Dishoeck, E. F., Bruderer, S., Pérez, L., \& Isella, A. 2015, A\&A, 579, A106

van Leeuwen, F. 2007, A\&A, 474, 653

Vorobyov, E. I. 2013, A\&A, 552, A129

Vorobyov, E. I., \& Basu, S. 2015, ApJ, 805, 115

White, R. L. 1979, ApJ, 229, 954

Whitney, B. A., Robitaille, T. P., Bjorkman, J. E., et al. 2013, ApJS, 207, 30

Zhu, Z., Dong, R., Stone, J. M., \& Rafikov, R. R. 2015, ApJ, in press (arXiv:1507.03599)

Zhu, Z., Nelson, R. P., Dong, R., Espaillat, C., \& Hartmann, L. 2012, ApJ, 755,6

Zhu, Z., Stone, J. M., Rafikov, R. R., \& Bai, X.-n. 2014, ApJ, 785, 122 